\title{
A suprasellar subarachnoid pouch; aetiological considerations
}

\author{
O BINITIE, BERNARD WILLIAMS, CP CASE \\ From the Midland Centre for Neurosurgery and Neurology, Smethwick, Warley, West Midlands, UK
}

SUMMARY A child with hydrocephalus treated by a valved shunt was reinvestigated after developing a shunt infection. A pouch was discovered invaginating the floor of the third ventricle and filling slowly with CSF from the region of the interpeduncular cistern. Histology and mechanisms of this pouch formation are discussed.

Arachnoid lined cysts in the subarachnoid space form about one percent of space occupying intracranial lesions in several series. ${ }^{1-3}$ These cysts may be separate from the normal subarachnoid space or may communicate with it. The term "cyst" may be applied to a fluid collection which has no macroscopic connection with other fluid containing space and "pouch" to a fluid collection with one entrance or exit. ${ }^{4}$ Cavities containing cerebrospinal fluid (CSF) occur throughout the neuraxis and may be in any of the layers, extradural, subdural, intraarachnoid or subpial. Such pouches have been described mostly in the supratentorial area with cerebral convexities and Sylvian regions predominating. ${ }^{25-11}$ Posterior fossa pouches, though less frequent, are well recognised.12-15 Arachnoid pouches in the hypophyseal region are even less common. ${ }^{1} 1617$ Suprasellar pouches which communicate with basal cisterns and intrasellar pouches communicating with extensions of the chiasmatic cistern have been described. ${ }^{16}$ The routine use of computed tomography (CT) has improved the diagnosis and management of these cases. The present case is of interest in having progressed despite treatment of an associated hydrocephalus and demonstration of contrast medium entering the pouch by sequential computed tomographic scanning.

\section{Case report}

Pregnancy and delivery at 39 weeks were normal in this boy, who had no serious illness until admission.

Address for reprint requests: Mr Bernard Williams, Midland Centre for Neurosurgery \& Neurology, Holly Lane, Smethwick, Warley, West Midlands B67 7JX, UK

Received 15 December 1983 and in revised form 29 March 1984. Accepted 1 April 1984
There was a family history of one sibling with spina bifida and two normal siblings aged four and six years. He was admitted to the Midland Centre for Neurosurgery and Neurology (MCNN) at the age of one and a half years because his head had been increasing in size over the previous six months. It was also noted that his arms and legs were stiff, that he did not attempt to crawl and his vocabulary was limited to basic words only. On examination, the child had a big head, (circumference $56 \mathrm{~cm}$ ) with a tight fontanelle. $\mathrm{He}$ was alert and appeared interested in his surroundings but with no intelligible speech. Tone was increased in all four limbs which moved reasonably well.

The attachment of the tentorium was seen to be low on lateral skull radiographs. Computed tomography (CT) showed a massive hydrocephalus with a small fourth ventricle (fig 1). On air ventriculography the aqueduct was shown to be patent because air passed to the fourth ventricle and cisterna magna.

On 22 June 1981 a right ventriculo-atrial shunt using a Holter valve was inserted. The child improved considerably and CT scans done in the subsequent weeks showed that the hydrocephalus was resolving but the third ventricle remained strangely wide. This was not commented upon at that time. The child continued to progress well and was able to crawl and say three words together prior to his second admission.

At the age of two and a half years, he was admitted again with malaise, loss of weight for a month, attacks of abdominal distension and occasional attacks of rigidity. On examination, he had a microcytic hypochromic anaemia, HB. $7 \cdot 3 \mathrm{~g} / \mathrm{dl}$ and hepatosplenomegaly. Bone marrow showed a myeloid and erythroid increase with megakaryocytes. There was no neck stiffness or other localising 
neurological feature. Ventricular CSF was sterile with white cells less than one per $\mathrm{mm} \cdot{ }^{3}$ Aspiration of CSF from the valve yielded staphylococcus epidermidis. The lower end of the valve tubing was drained externally. The child was treated with antibiotics and improved.

CT scanning showed that the third ventricle was globular and much enlarged despite the lessening of the hydrocephalus. Cisternal amipaque studies showed that the contrast medium travelled up the back of the clivus readily but stopped below the posterior clinoid process and despite keeping the child in a head down position for many minutes did not appear to enter the region of a presumed pouch (fig 2). The immediate CT scans showed the globular nature of the pouch clearly and showed that contrast medium spread up behind it in the subarachnoid spaces (fig 3). The CT scans were repeated after some hours and these showed that although contrast material had not entered the ventricles some had entered the pouch (fig 4).

Combined ventricular and lumbar pressure studies were carried out with $3 \frac{1 / 2 "}{2}(8.75 \mathrm{~cm}) 18 \mathrm{G}$ lumbar puncture needles in a lateral ventricle and the lumbar spinal theca. Despite the investigation being done under local anaesthetic and the child coughing and occasionally struggling during the test-

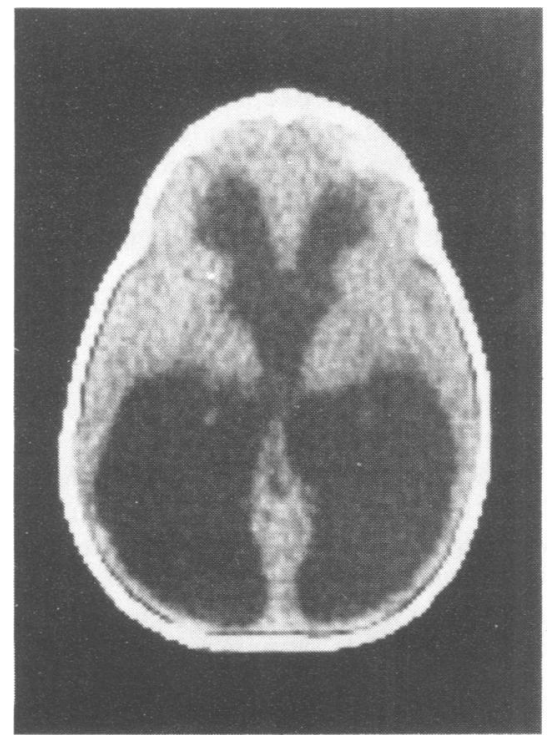

Fig 1 CT scan including the third ventricle at the time of presentation. The third ventricle in common with other midline structures is often compressed in the early stages of hydrocephalus although later on it commonly becomes enlarged. This third ventricle is therefore not certainly abnormal.

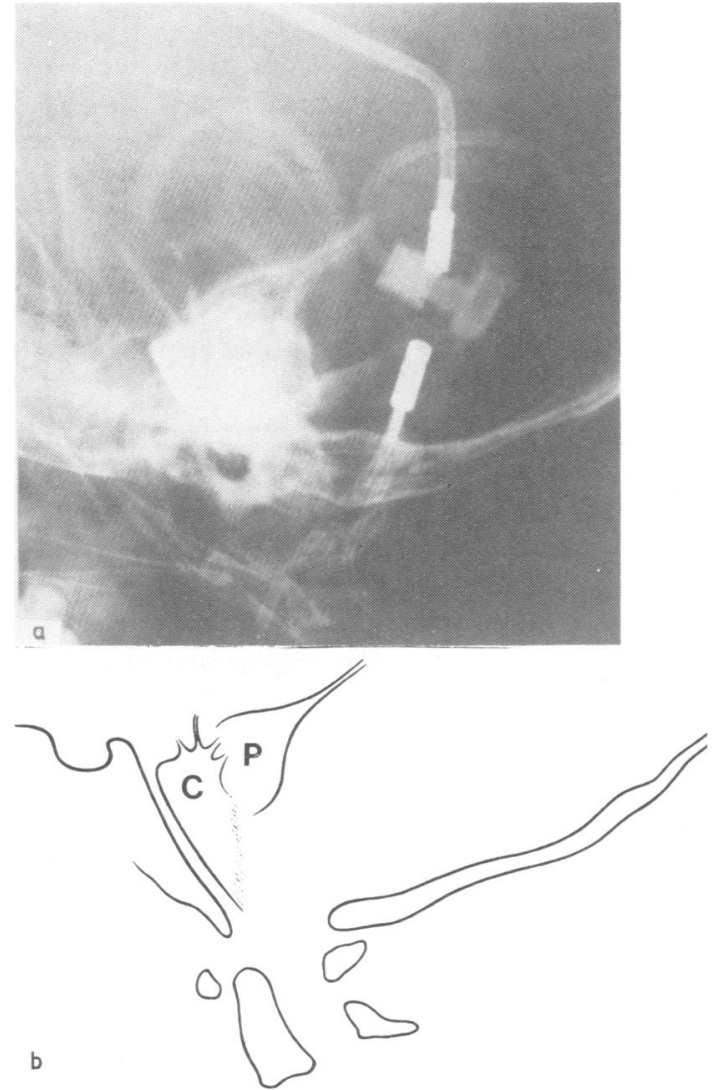

Fig 2 (a) Lateral radiograph, prone, head down after injection of water soluble contrast medium into the cisterna magna. There is arrest of upward flow of the medium at the level of Liliequist's membrane. (b) Key to fig $2 a$. C is the location of contrast medium in the cisterna pontis, $P$ is the petrous bones. Note the streaks of medium which appear to be attempting to move rostrally.

ing the pressures were substantially equal in both compartments throughout and no mechanism suggesting inflation of the pouch in the head similar to that reported by Al-Din and Williams ${ }^{4}$ was found.

At right frontal craniotomy a large fluid collection replacing the third ventricle was seen through a circular incision made into the right frontal lobe. There was a thin domed roof to the pouch which protruded upwards through the site of a greatly enlarged foramen of Munro. The ventricular CSF had a total protein of $0.08 \mathrm{~g} / \mathrm{l}$, that from the pouch was closer to lumbar CSF values with a level of $0.28 \mathrm{~g} / \mathrm{l}$. Chloride level was $117 \mathrm{mmol} / \mathrm{l}$ in each, the sugar content was $3.3 \mathrm{mmol} / \mathrm{l}$ in the pouch and $3.6 \mathrm{mmol} / \mathrm{l}$ in the ventricular fluid. The pouch roof was largely excised as 

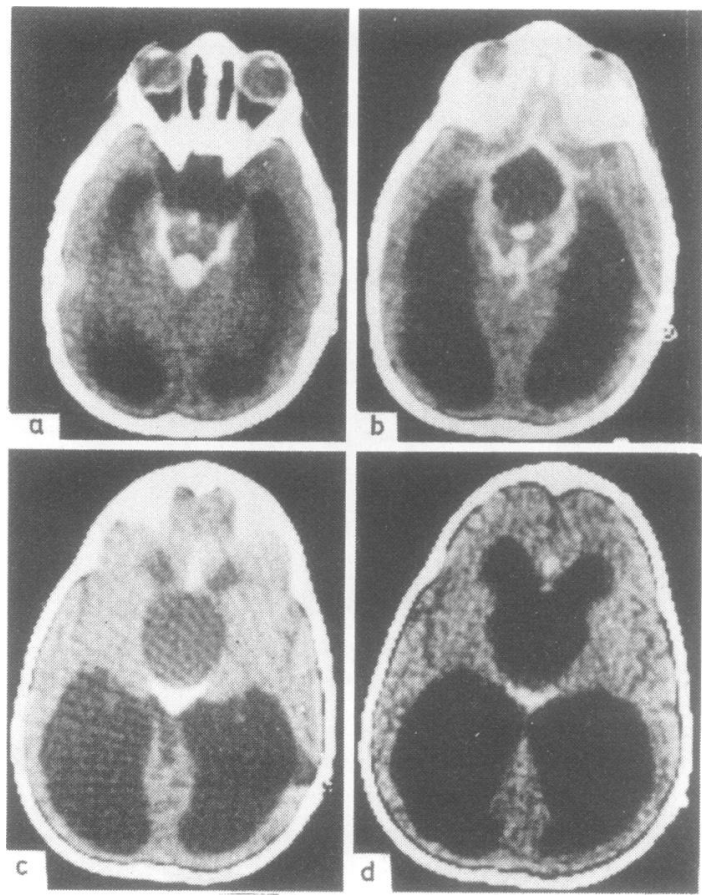

Fig 3 CT scans of the basal regions shortly after the cisternogram shown in fig 2. (a) The cisterns are well shown leading the contrast medium dorsally to the back of the hindbrain (cisterna ambiens). (b) Contrast medium is spreading around the pouch along the course of the main cerebral vessels but the pouch is not filling readily. (c) The rotundity of the pouch is well seen together with its anatomical separateness from the frontal horns. Contrast medium here behind the pouch is in the region of the tela choroidea, that is above the devarication of the brainstem into the two hemispheres. (d) The size of the pouch is well seen in this section and the septum dividing it from the lateral ventricles is not seen. The shape of the third ventricle with its globular outline is the clue to the diagnosis in this section.

a biopsy, revealing normal pia covering the medial edges of the temporal lobe below. The infected shunt was removed and a left sided ventriculoperitoneal shunt with a Hakim-Cordis valve was inserted. After the operation CT scans showed shrinkage of the pouch and a reduction in the size of the ventricles. His progress was satisfactory and he was discharged on 20.7.82.

\section{Histology}

The biopsy was fixed in buffered formal saline for light microscopy. Small pieces were postosmicated in $1 \%$ osmium tetroxide in $0.1 \mathrm{M}$ Phosphate buffer, for electron microscopy.

Routine paraffin sections stained with haematoxy-

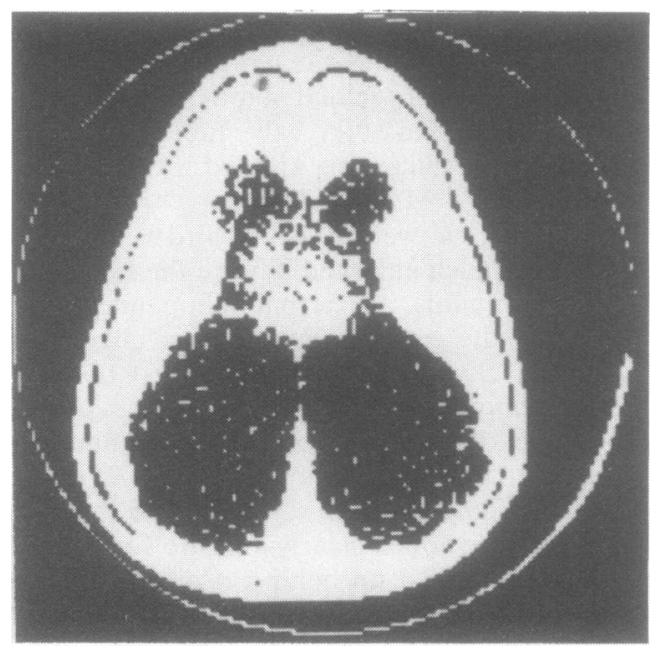

Fig 4 CT scan four hours after the cisternogram. The greater part of the pouch is opacified to a value of eleven Hounsfield units indicating that contrast medium has entered the pouch.

lin and eosin showed the top surface to be covered partly by cuboidal or columnar ependymal cells with recognisable blepharoplasts, but with considerable areas of more flattened cells without blepharoplasts. The inferior surface was covered by arachnoidal cells, sometimes several cells thick. In sections stained with Massons Trichrome (fig 5) the wall consisted of a glial layer deep to the ependymal cells and of dense collagen bundles deep to the arachnoid layer with a looser and more vascular central zone. Within the glial layer were occasional recognisable, but degenerate neurons and small clumps of ependymal cells together with moderate numbers of astrocytic nuclei. The collagen of the middle looser layer interdigitated closely with the glial layer in a similar manner to the pia over normal brain.

The ultrastructural appearances showed that both the ependymal cells on the top surface (fig 6) and those buried within the subependymal glia were morphologically similar to those which have been described in normal brain. ${ }^{18} 19$ Furthermore, the ependymal cells on the top surface contained numerous microvilli with only single groups of cilia, which corresponds to scanning electron microscopical observations of the floor of the normal human third ventricle..$^{20}$ The adjacent flattened cells on the top surface were more separated than the ependymal cells and may have been astrocytes or modified ependymal cells as seen after hydrocephalus. ${ }^{21}$ The ependymal elements were not divisible into ciliated cells and non-ciliated cells which contained secretory material or which showed an electron dense 


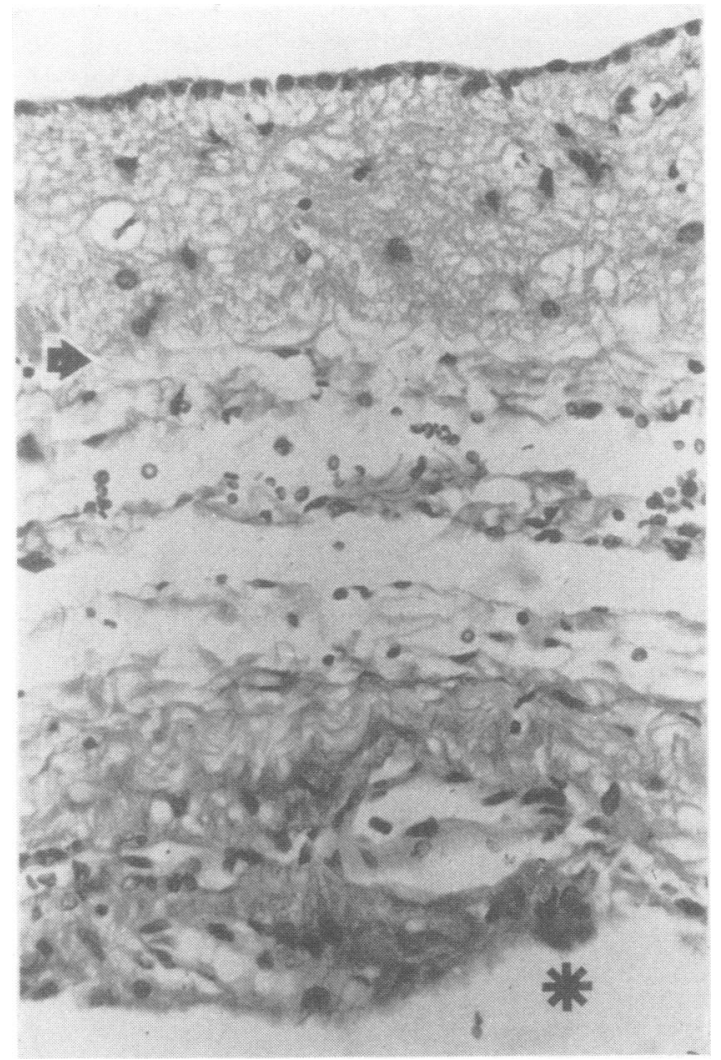

Fig 5 Paraffin section stained with Masson Trichrome shows at the top an almost continuous ependymal layer over glial tissue and at the bottom the arachnoidal layer with grouped $\left({ }^{*}\right)$ arachnoidal cells. The middle looser layer

(\$) shows interdigitating glia and loose collagen, similar to the pia over normal brain (magnification $\times 125$ ).

coating to their microvilli. Nor were they generally separated from the subependymal glia by basement membrane although a network of basement membranes similar to that described by Leonhardt and Desaga $^{22}$ was found between superficial and buried ependymal cells, flattened cells and glia (for example in fig 6). The ependymal cells were, therefore, morphologically different from the cells which line colloid cysts $^{23}$ or to a varying extent from epithelial cysts in various sites. ${ }^{24-26}$

\section{Discussion}

The histological appearance of the roof of the pouch was that of the floor of the third ventricle which had been subjected to increased pressure rather than that of a congenital cyst and it must be assumed that the force responsible for the pouch must have been generated from below the third ventricle floor rather than within it.

Brocklehurst $^{27}$ has described cystic cavities in this situation. He opined that what he termed diencephalic cyst was probably a maldevelopment present before the $60 \mathrm{~mm}$ stage due to failure of the diencephalon to sink ventrally after the anterior neuropore had closed. Associated maldevelopments may occur such as agenesis of the corpus callosum or other midline cysts. The mechanism of the associated hydrocephalus was difficult to understand. Brocklehurst's cases, however, are obviously not similar to ours and, because of the associated maldevelopments in his cases it seems reasonable to regard them as entirely different. It appears unlikely, for example, that localised changes in CSF pressure could be responsible for the deformities described by Brocklehurst. The arachnoid pouches under consideration occur however, with fundamentally normal brains which are merely distended by the expansion of the pouch or an associated hydrocephalus.

Cases of symptom-free arachnoid pouches have been discovered accidentally in the course of an investigation or at necropsy. ${ }^{28-30}$ Thus a large arachnoid pouch may remain silent during life or may produce intermittent symptoms and signs. The most common presentations are of raised intracranial pressure, optic nerve or brain stem compression and pituitary or hypothalamic dysfunction. The "bobble-headed doll" syndrome is amongst the most interesting presentations associated with central suprasellar arachnoid pouches. This was reported by Benton et al in 1966 as a syndrome of trunkal tremor associated with third ventricular cyst and hydrocephalus in children. ${ }^{31}$ The patho-physiology is not known although other cases have been reported and discussed. ${ }^{32}{ }^{33}$ Russo and Kindt suggested that the enlarged third ventricle exerted pressure on the dorso-medial nucleus of the thalamus and that this was responsible for the to and fro head movement. ${ }^{34}$

Pouches in relation to the subarachnoid space may cause distortion and appear to be acting as an obstruction to CSF pathways in much the same way as other space occupying lesions. Alternatively, the pouch may be an associated phenomenon only, that is to say, neither the cause nor the result of the hydrocephalus but a feature due to the same cause, for instance, abnormal arachnoid bands or adhesions or some generalised defect of development. They are uncommon lesions compared with hydrocephalus, nevertheless, it is possible that the high pressure associated with the hydrocephalus may play a part in the pathogenesis. Symptoms from such pouches may tend to progress rapidly once manifest and though no neoplastic change has occurred in the cyst wall, it may still expand. Various explanations 


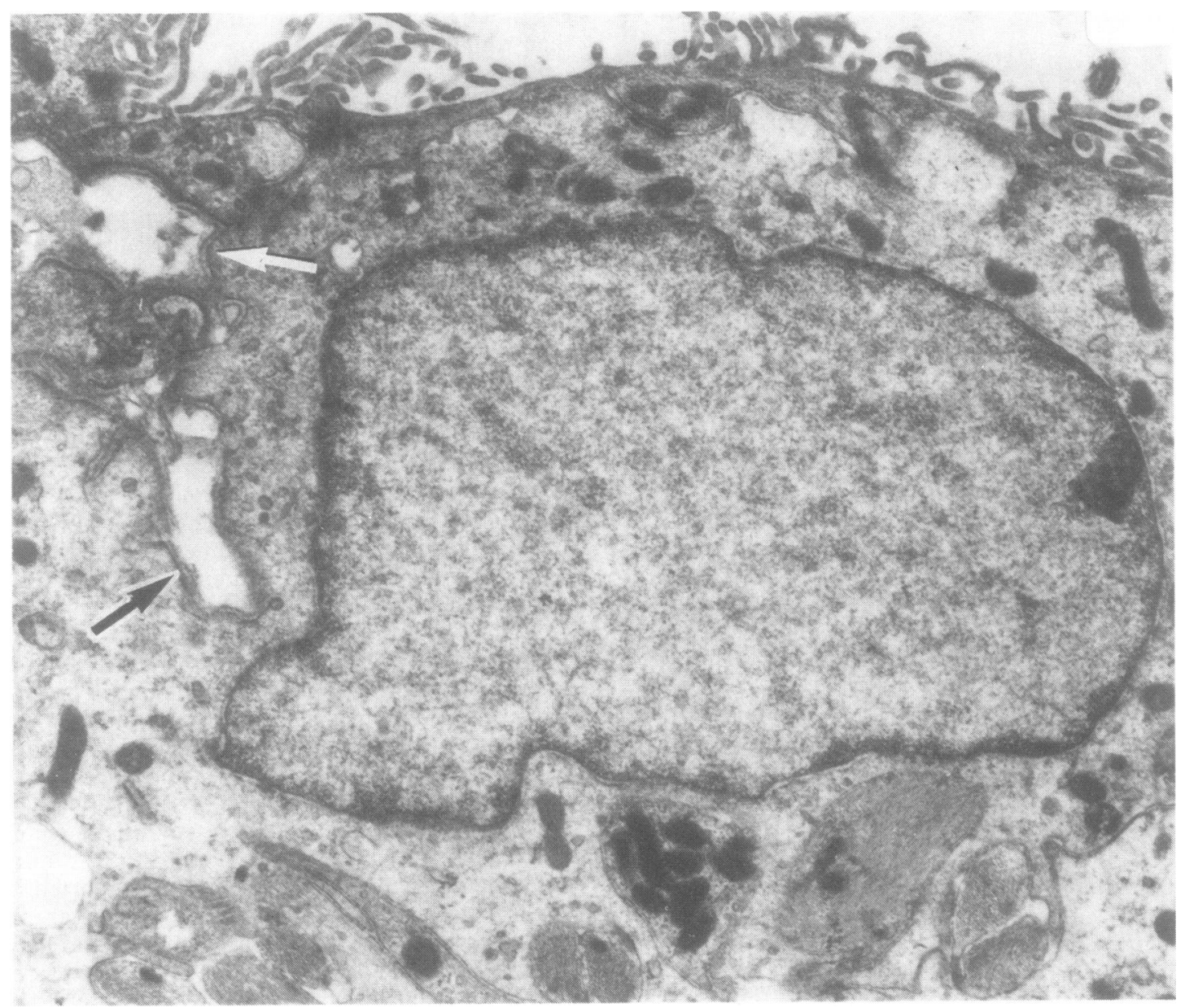

Fig 6 Electron micrographs of an ependymal cell from the top surface which rests directly on the subependymal glia. A rare example of extracellular space between ependymal cells which is lined by basement membrane is shown (arrows) and is associated with ependymal cell membrane thickening. In general the extracellular spaces which were lined by basement membrane were not specifically distended (magnification $\times 8,600$ ).

have been given to account for this expansion.

In review of theories of arachnoid cyst enlargement Krawchenko and Collins ${ }^{28}$ included a "ball valve" action between the cyst and the subarachnoid space, an osmotic gradient, "pulsating pressure waves in the CSF and along blood vessels", and transudation from choroid plexus tissue remnants or ectopic glial cells. This assortment of unrelated possibilities do not reflect these observed facts or simple physical principles.

Firstly, osmosis may be disposed of because the fluid in the pouches is indistinguishable from CSF. If it is not, then such a cyst is not in the class of structures under discussion. Secondly, ectopic glial cells seem to be an unlikely cause. There are glial cells all over the CNS, they do not cause CSF containing pouches in such sites so why should they do so when they are ectopic? The description by Cooper and Kernohan $^{35}$ of glial nests in the meninges has been passed on in the literature following the publication of Jakubiak, Dunsmore and Beckett as though this were an explanation of subarachnoid pouch formation that demanded serious consideration. ${ }^{10}$ Next to consider is "choroid plexus remnants". The secretory force of choroid plexus may be responsible for hydrocephalus and for the distension of parts of the ventricular system which become pinched off such as a trapped temporal horn. In the same way it may be implicated in varieties of "Dandy-Walker cyst" where there is choroid plexus within an abnormally 
distended cavity. It is, however, notable that in the majority of CSF-containing pouches there is no choroid plexus, and in the presence of a pouch with proven communication to the subarachnoid space, such as in the present patient, there is no possibility of choroid plexus secretion distending a localised section unless it is closed off from the remainder of the pathways by a valvular mechanism. If there is a valve and a filling mechanism then the choroid plexus is an unnecessary postulate.

It seems evident that in the case of a pouch of this kind a valvular mechanism must be involved. The term "ball valve" is often used. ${ }^{2 \times 36}$ The most ball like structures in the CNS are colloid cysts and spinal neurofibromata, the latter sometimes impact across the spine after lumbar puncture. Colloid cysts in the anterior third ventricle are often round and tense but are usually tightly in amongst surrounding tissues and unable to move as a ball valve. A better example of a bulk of tissue acting as a valve is the whole of the hindbrain which may move up and down and intermittently jam into the foramen magnum. Moulding of the structures into a hindbrain hernia or Chiari type I malformation and the efficacy of this shape as a valve has been documented $^{37-34}$ and an association with posterior fossa pouches has been described. ${ }^{40}$ In these cases it is likely that the mouth of the pouch is held closed by a plug of tissue as soon as the pressure above the foramen magnum is higher than that in the spine.

The only requirement for a valvular mechanism to be present is that pressure on the high side of the system tends to hold the entrance closed and thus to maintain at least a temporary pressure difference. A more frequent and more relevant kind of valve occurs as illustrated in fig 7. A partly inflated toy balloon can illustrate such a valve mechanism by placing it on a hard surface and rolling the inflated body over the neck of the balloon. The presssure is equal in the balloon and the neck as this is done; the greater area of the body, however, means that a greater force is brought to bear downwards upon the neck than that exerted by the pressure within it. Elastic forces in the wall of the balloon also play a part and it is difficult to make a balloon stay inflated in this way. Nevertheless, the emptying is greatly delayed and if spasmodic refilling was available such a simple model might well represent the pouches under discussion ( $\mathrm{fig} 7$ ). If a situation similar to such a balloon were found in the brain and a similar cyst was opened by a neurosurgeon, he would find just a slit in the bottom. If he were to try to describe it he might use the term slit or flap valve. ${ }^{3041}$ If CSF could not be persuaded to flow into it at operation such a communication might be difficult to demonstrate.

The siting of such a morphological oddity may be expected to occur at points where pre-existing narrowings occur and also where there are firm surfaces. Thus, the incisura is a natural site and both the clivus and the edges of the tentorium provide such a surface. The foramen magnum is another common site and in some cases the falx cerebri" or the dura of the spinal cord may provide counter pressure during the formation of valvular pouches. ${ }^{42}$

It was suggested by Fox and Al-Mefty that suprasellar arachnoid pouches sometimes began as a diverticulum of a variable sheet of arachnoid across the region of the interpeduncular cistern running from approximately the position of the oculomotor nerves or the edge of the incisura, across to the opposite side. ${ }^{30}$ This structure is commonplace to neurosurgeons who approach the incisura with the microscope either from below for trigeminal nerve root section or from above. It commonly seems to be attached to the carotid artery or to the arachnoid around it, and may be broken through while dissecting a posterior communicating artery position aneurysm. Fox and Al-Mefty illustrate an attachment to the posterior rim of the sella anteriorly and to the mammillary bodies above. They note that this zone of tissue was described by Key and Retzius, although they prefer to call it the membrane of Liliequist. They further comment that the membrane is normally perforated or otherwise permeable to CSF.

There is little merit in postulating that these tissues need to be an anatomically recognisable structure. Liliequist himself found temporary hold up of air in only $25 \%$ of 120 air encephalograms at this site, and in $17 \%$ of these he did not find a convex shape corresponding to this membrane..$^{29}$ Occlusion of this membrane by deposition of adhesions in its vicinity with additional webs of arachnoid as a result of haemorrhage, infection or some variety of maldevelopment might encourage it to entrap upward pulsations and thus to be expanded forward and upward into the floor of the third ventricle. Thickening and excess of such adhesions may sometimes be due to birth injury or other head injury. ${ }^{4043}$ Many authorities refer to "primary" or "secondary" cysts implying that infection or trauma cause the secondary kind of pouch and therefore that there is a difference in mechanism between such cases. This distinction further implies that the primary kind is "developmental". There does not seem to be any value in such a distinction other than from that kind of gross embryonic disorganisation mentioned earlier in association with the cases of Brocklehurst. Of more importance is a consideration of what causes the simple pouches under discussion to fill. Plainly an energetic filling mechanism must be responsible.

Pulsatile energy transmitted from the arteries 

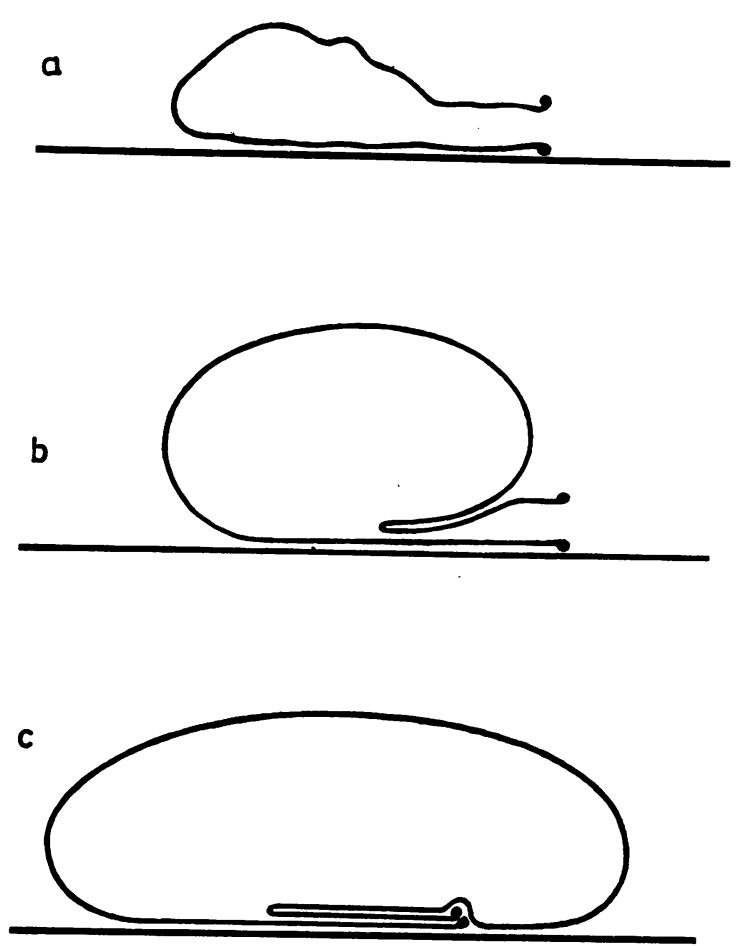

Fig 7 Proposed sequence of events in the formation of pouches of this kind as illustrated by the use of a toy balloon. (a) Any distension of the balloon with a fully open neck leads to rapid equalisation of pressure after intermittent inflation. (b) If the distension of the balloon is such that the neck becomes narrowed by the expansion of the body, then the intermittent forces causing distension become more effective and enduring, the body of the balloon may become still larger and cause progression to the next stage. (c) Substantial compression of the filling route by the body of the balloon may lead to a situation in which the neck of the balloon acts as a valve with a high degree of competence. Pulses of fuid pressure which fill the structure may have difficulty in escaping.

might, it has been suggested, provide a pumping action for CSF. ${ }^{16}$ This concept has been criticised because very little alteration occurs in intracranial arterial diameters or lengths during systole; in addition, there would have to be some remarkable perivascular anatomical features to allow localised increase in pressures in the CSF outside the arteries, and even if such localised pressure could be transiently produced it seems unlikely to be generated sequentially along a flow path thus guiding fluid through the neck of a pouch. ${ }^{4546}$

The contours of the subarachnoid space were described as pliant and very responsive to changes in
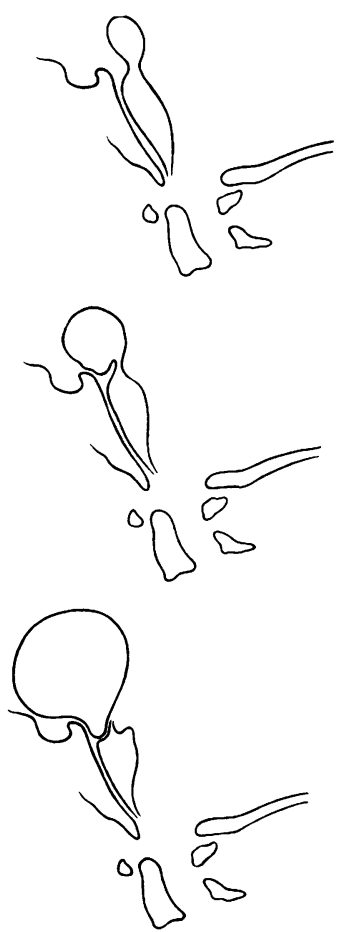

Fig 8 Proposed sequence of changes analagous to the stage of balloon distension in fig 7. (a) Upward distension of part of the membrane of Liliequist, capped by the floor of the third ventricle, might be commonplace in hydrocephalus and be difficult to detect if the neck was uncompressed and deflation took place easily. (b) Continued expansion of such a pouch might lead to narrowing of the neck and exaggerate the progression of the pouch. (c) A pouch of this size may be inert, both difficult to inflate and to deflate. For it to continue to exist however there must be at least a slow or intermittent filling from the subarachnoid space cf figs 2 and 4.

venous pressure in $1935 .{ }^{47}$ Williams recorded venous pulsations of over $100 \mathrm{~mm} \mathrm{Hg}$ amplitude and almost equal CSF pressure changes in response to venous influences during coughing. Pressure gradients in CSF of $10-20 \mathrm{~mm} \mathrm{Hg}$ were recorded between the lower and upper ends of the spinal subarachnoid space during the initial rise which followed a cough. ${ }^{39}$ Du Boulay recorded CSF movement of only $0 \cdot 1 \mathrm{ml}$ along the aqueduct per cardiac cycle, whereas coughing and straining shifted 2-9 ml CSF into the cranial cavity. ${ }^{456}$ Repeated alterations in venous pressure resulting in CSF waves are thus many times more likely to provide the pulsatile energy which moves CSF under high pressure and thereby expands arachnoid pouches, than the pressures transmitted to CSF by arteries. Furthermore the most energetic part of such pulsation is directed 
upwards in the correct direction to fill a pouch such as that illustrated in fig 8 .

The relationships between a hydrocephalus and a subarachnoid pouch may be complex. It is not justifiable to conclude that such pouches cause the hydrocephalus. ${ }^{13}$ Firstly, whatever causes one may cause the other, if there are adhesions and webs of fibrous tissue occupying the CSF pathways at the base of the brain secondary to meningitis for instance; such obstructions might contribute to both features. Secondly, the hydrocephalus, if it precedes the formation of the pouch may predispose to its formation in several ways. A pouch like the present one may be regarded as an inpushing of the floor of the third ventricle; the distension and backward protrusion of the floor of the third ventricle downwards through the incisura in the early stages of a hydrocephalus may well provide a weakening and expansion of that part of the wall as a precursor of later inversion. Another important morphological effect of hydrocephalus is to narrow the CSF channels at the inciscura. Not only the floor of the third ventricle but also the suprapineal recess and the temporal horns tend to become jammed into the incisura, narrowing the pathways available and perhaps increasing the likelihood of a valve being formed. Similar narrowing at the foramen magnum may also be increased by an associated hydrocephalus and downward herniation of the tonsils.

An additional hydrodynamic mechanism by which hydrocephalus may predispose towards such pouches concerns the state of filling of the veins. If the overall CSF pressure within the neuraxis is high then, in the relaxed subject, the majority of collapsible veins including the epidural veins are flattened. This means that a sudden rise in thoraco-abdominal pressure such as is caused by a cough is accompanied by the displacement of a greater volume of blood into the spine than if the veins were partly prefilled. ${ }^{34}$ Another effect of high pressure and flattening of veins is that the capacitance or compliance of the neuraxis is diminished and an influx of venous blood produces a higher pressure pulse than in the normal. With both the movement and the pressure increased above normal therefore the energy transmitted to the CSF pathways may be markedly increased and thus the development of a pouch-like cavity is more likely. ${ }^{38}$

A further feature is the likelihood that the pouch may block the CSF pathways, either at the incisura or the aqueduct. Provided that the pressure within the pouch is higher than that within the ventricles, which presupposes a valve and a suitable filling mechanism, such an occurrence is possible although it may be intermittent.

Direct attack upon the pouch either along the base of the skull or through a lateral ventricle is not always successful in controlling the hydrocephalus. Treatment of the hydrocephalus by a valved extrathecal shunt alone also may be unsuccessful. A similar case reported earlier $r^{4}$ and treated only by a valved extrathecal shunt has subsequently deteriorated requiring further operation on an expanding pouch.

In the state of uncertainty surrounding the interrelationship between the pouches and hydrocephalus it would seem sensible to treat both of the conditions at the same time as was done with this case, opening the top of the pouch and putting in a valved shunt. An interesting suggestion came from $\mathrm{Kasdon}^{48}$ that after a valved shunt had been placed in the ventricles a separate tube could be left from the pouch to the subscalp area for later conversion to a drain if required. Careful pressure studies may elucidate the mechanisms further in the future although they appear to be unnecessary for adequate treatment to be performed.

The authors thank Dr. Michael V. Salmon for helpful discussions and Mr. Derek Webb for help with the electron microscopy.

\section{References}

' Dott NM, Gillingham FJ. Mechanical aspects of the cerebrospinal fluid circulation-physiological, pathological, surgical. In. The Cerebrospinal Fluid. 1958;246-64.

2 Starkman SP, Brown TC, Linell EA. Cerebral arachnoid cysts. J Neuropath Exp Neurol 1958;17:484-500.

${ }^{3}$ Robinson RG. Congenital cysts of the brain: Arachnoid malformations. Prog Neurol Surg 1971;4:133-74.

${ }^{4}$ Williams B, Guthkelch AN. Why do central arachnoid pouches expand? J Neurol Neurosurg Psychiatry 1974;37: 1085-92.

s Aicardi J, Nauman F. Supratentorial extracerebral cysts in infants and children. $J$ Neurol Neurosurg Psychiatry. 1975;38:57-68.

- Anderson FM, Landing BH. Cerebral arachnoid cysts in infants. J Pediatr 1966;69:88-96.

${ }^{7}$ Bhandari YS. Non-communicating supratentorial subarachnoid cysts. J Neurol Neurosurg Psychiatry 1972;35:763-70.

× Dyck P, Gruskin P. Supratentorial arachnoid cysts in adults. A discussion of two cases from a pathophysiologic and surgical perspective. Arch Neurol 1977;34:276-9.

${ }^{9}$ Al-Din A, Williams B. A case of high pressure intracerebral pouch. J Neurosurg Psychiatry 1981;44:918-23.

${ }^{10}$ Jakubiak P, Dunsmore RH, Beckett RS. Supratentorial brain cysts. J Neurosurg 1968;28:129-36.

"Harrison MJG. Cerebral arachnoid cysts in children. $J$ Neurol Neurosurg Psychiatry 1971;34:316-23. 
12 Trowbridge WV, French JD. Benign arachnoid cysts of the posterior fossa. J Neurosurg 1952;9:398-404.

${ }^{13}$ Alvord EC, Marcuse PM. Intracranial cerebellar meningoencephalocele (posterior fossa cyst) causing hydrocephalus by compression at the incisura tentori. J Neuropath Exp Neurol 1962;21:50-69.

${ }^{14}$ Matson DD. Neurosurgery of Infancy and Childhood. 2nd Ed. Springfield: Thomas, 1969.

is Di Rocco C, Caldarelli M, Di Trapam G. Infra-tentorial arachnoid cysts in children. Child's Brain 1981;8:119-33.

${ }^{16}$ Banna M. Arachnoid cysts in the hypophyseal area. Clin Radiol 1974;25:323-6.

${ }^{17}$ Danziger J, Bloch S. Suprasellar arachnoid pouches. Br J Radiol 1974;47:448-51.

${ }^{18}$ Brightman MW, Palay SL. The fine structure of ependyma in the brain of the rat. $J$ Cell Biol 1963; 19:415-39.

${ }^{19}$ Blakemore WF, Jolly RD. The subependymal plate and associated ependyma in the dog. An ultrastructural study. J Neurocytol 1972;1:69-84.

${ }^{20}$ Bannister CM, Chapman SA. Ventricular ependyma of normal and hydrocephalic subjects: A scanning electron-microscopic study. Develop Med Child Neurol 1980;22:725-35.

${ }^{21}$ Weller RO, Wiśniewski H, Shulman K, Terry RD. Experimental hydrocephalus in young dogs. Histological and ultrastructural study of the brain tissue damage. J Neuropath Exp Neurol 1971;11:613-26.

${ }^{22}$ Leonhardt H, Desaga U. Recent observations on ependyma and sub-ependymal basement membranes. Acta Neurochir 1975;31:153-9.

${ }^{23}$ Hirano A, Ghatak NR. The fine structure of colloid cysts of the third ventricle. J Neuropath Exp Neurol 1974;33:333-41.

${ }^{24}$ Hirai O, Kawamura J, Fukumitsu T. Prepontine epithelium-lined cyst. Case report. J Neurosurg 1981;55:312-7.

${ }^{25}$ Hirano A, Ghatak NR, Wisoff HS, Zimmerman HM. An epithelial cyst of the spinal cord. An electron microscopic study. Acta Neuropath (Berl) 1971;18:214-33.

${ }^{26}$ Pearl GS, Takei Y, Tindall GT, Hoffman JC. Supratentorial subarachnoid epithelial cyst: case report and ultrastructural study. Neurosurgery 1982;11:48-51.

${ }^{27}$ Brocklehurst G. Diencephalic cysts. J Neurosurg 1973;38:47-51.

${ }^{28}$ Krawchenko J, Collins GH. Pathology of an arachnoid cyst. J Neurosurg 1979;50:224-8.

${ }^{29}$ Liliequist B. The subarachnoid cisterns: An anatomic and roentgenologic study. Acta Radiol (suppl) (Stockh) 1959;185:1-108.

30 Fox JL, Al-Mefty. Suprasellar Arachnoid Cysts: An extension of the membrane of Liliequist. Neurosurgery 1980; 7:615-8.

${ }^{31}$ Benton JW, Nellhaus G, Huttenlocher PR, Ojemmann RG, Dodge PR. The Bobble-head doll syndrome. Neurology (Minneap) 1966;16:725-9.

${ }^{32}$ Nellhaus G. The Bobble-head doll syndrome: "Tic" with a neuropathologic basis. Paediatrics 1967;40:250-53.

${ }^{33}$ Dell S. Further observations on the "Bobble-headed Doll Syndrome". J Neurol Neurosurg Psychiatry 1981;44:1046-9.

${ }^{34}$ Russo RH, Kindt GW. A neuroanatomical basis for the Bobble-head Syndrome. J Neurol 1974;41:720-3.

${ }^{35}$ Cooper IS, Kerhohan JW. Heterotopic glial nests in the subarachnoid space; histopathic characteristics, mode of origin and relation to meningeal gliomas. $J$ Neuropath Exp Neurol 1951;10:16-29.

${ }^{36}$ Hoffman HJ, Hendrick EB, Humphreys RP, Armstrong EA. Investigation and management of suprasellar arachnoid cysts. J Neurosurg 1982;57:597-602.

${ }^{37}$ Williams B. Combined cisternal and lumbar pressure recordings in the sitting position using differential manometry. J Neurol Neurosurg Psychiatry 1972;25: 142-3.

${ }^{38}$ Williams B. A demonstration analogue for ventricular and intraspinal dynamics. J Neurol Sci 1974;23: 445-61.

${ }^{39}$ Williams B. Cerebrospinal fluid pressure changes in response to coughing. Brain 1976;99:331-46.

${ }^{40}$ Williams B. Subarachnoid pouches of the posterior fossa with syringomyelia. Acta Neurochir 1979;47:187-217.

${ }^{41}$ Kasdon DL, Douglas EA, Brougham MF. Suprasellar arachnoid cyst diagnosed preoperatively by computerised tomographic scanning. Surg Neurol 1977;5:299-303

${ }^{42}$ McCrum C, Williams B. Spinal extradural pouches. $J$ Neurosurg 1982;57:849-52.

${ }^{43}$ Contreras C, Copty M, Langelier R, Gagne F Traumatic suprasellar arachnoid cyst. Surg Neurol 1977;3:196-8.

${ }^{44}$ Williams B, Guthkelch AN. Expansion of central arachnoid pouches. Child's Brain 1975;1:364-7.

${ }^{4 s} \mathrm{Du}$ Boulay GH. Pulsatile movements in the CSF pathways. Br J Radiol 1966;39:255-62.

${ }^{46}$ Du Boulay GH, O' Connell JE, Currie JEA, Bostock J, Verity $P$. Further investigations "on pulsatile movements in the cerebrospinal fluid pathways. Acta Radiol 1972; 13:469-523.

${ }^{47}$ Bedford THB. The effect of increased intracranial venous pressure on the pressure of the cerebrospinal fluid. Brain 1935;58:437-47.

${ }^{4 x}$ Kasdon DL. Discussion of paper by Fox and Al Mefty Neurosurgery 1980; 7:618. 